\title{
Photoelimination of Nitrogen from Diazoalkanes: Involvement of Higher Excited Singlet States in the Carbene Formation
}

\author{
Tomislav Piteša, ${ }^{\ddagger}$ Marija Alešković, ${ }^{\prime}$ Kristin Becker, ${ }^{\|}$Nikola Basarić, ${ }^{\prime *}$ and Nađa Došlić ${ }^{* *}$ \\ ¥Department of Physical Chemistry, Ruđer Bošković Institute, Bijenička cesta 54, 10000 Zagreb, Croatia. \\ ' Department of Organic Chemistry and Biochemistry, Ruđer Bošković Institute, Bijenička cesta 54, 10000 Zagreb, Croatia. \\ KEYWORDS Diazoalkanes, Carbenes, Anti-Kasha Photochemistry
}

\begin{abstract}
Although diazoalkanes are important carbene precursors in organic synthesis, a comprehensive mechanism of photochemical formation of carbenes from diazoalkanes has not been proposed. Synergy of experiments and computations demonstrates the involvement of higher excited singlet states in the photochemistry of diazoalkanes. In all investigated diazoalkanes, excitation to $\mathrm{S}_{1}$ results in nonreactive internal conversion to $\mathrm{S}_{0}$. On the contrary, excitation to higher-lying singlet states $\left(\mathrm{S}_{\mathrm{n}}, \mathrm{n}>1\right)$, drives the reaction toward a different segment of the $S_{1} / S_{0}$ conical intersection seam and results in nitrogen elimination and formation of carbenes.
\end{abstract}

\section{Introduction}

Photoreactions taking place from higher excited states, known as anti-Kasha photochemistry, ${ }^{1}$ are rare since not many photochemical processes can compete with fast internal conversion (IC) to the first excited electronic state $\left(\mathrm{S}_{1}\right)$. Notable examples involve formation of nitrenes by elimination of nitrogen from higher excited singlet states $\left(\mathrm{S}_{\mathrm{n}}\right)$ of the corresponding azides in 4-amino-3-nitrophenyl azide, ${ }^{2}$ or 2-naphthoyl azide. ${ }^{3}$ However, anti-Kasha reactivity is not generally observed for all azides. For instance, 1-naphthyl azide reacts from $\mathrm{S}_{1}$ due to a low energy barrier toward arylnitrene formation in that state, while the $\mathrm{S}_{2}$ state is not reactive. ${ }^{4}$ Understanding ways to exploit the antiKasha reactivity has fundamental and technological value as it offers opportunities for a clever design of different photochemical processes where the selectivity of a reaction can be tuned by an appropriate choice of the excitation wavelength.

In this work we focus on diazoalkanes 1-3 (Chart 1) and demonstrate experimentally and computationally the general importance of higher excited singlet states in their photochemistry and photophysics. Diazoalkanes are versatile reagents in organic synthesis, ${ }^{5}$ which can undergo thermally or photochemically induced elimination of nitrogen to give carbenes. ${ }^{6}$ Over the years, photo-generated carbenes have been used for the methylation of carboxylic acids, alcohols, phenols, heteroatoms including nitrogen and sulfur, cyclopropanations, ring expansions and cycloadditions. ${ }^{7}$ Photochemistry is particularly interesting since spatial and temporal control over the formation of carbenes may pave the road to applications in biological systems. ${ }^{8,9}$

Previous experimental studies were concerned with larger diazoalkanes, particularly diphenyl derivative 3 due to weaker explosive character and easy excitation. The chemistry of carbene car-3 has been completely unraveled, ${ }^{10-23}$ but not much has been learned on the photochemical elimination reaction. From the computational side, an early CASSCF study suggested the pos- sibility of a wavelength dependent $\mathrm{CH}_{2}+\mathrm{N}_{2}$ formation in diazirine, ${ }^{24}$ but this possibility was subsequently dismissed. ${ }^{25}$ The existence of an extended seam of conical intersections (CI) along the $\mathrm{C}-\mathrm{N}$ dissociation coordinate was revealed in diazo compounds undergoing photoinduced Wolff rearrangement, and segments of the seam were associated with different photoproducts. ${ }^{26,27}$ These findings, however, were not positioned in the more general context of anti-Kasha reactivity.

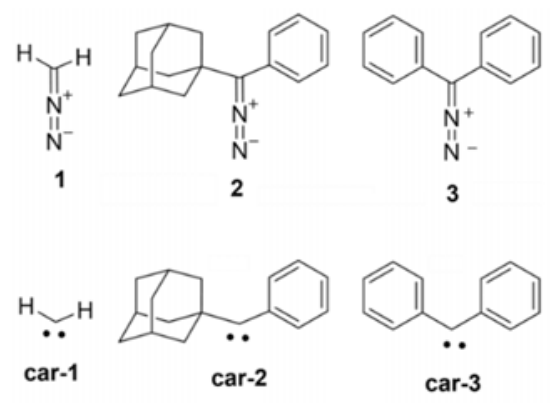

Chart 1. Diazoalkanes 1-3, and the corresponding carbenes car-1car-3.

\section{Results and Discussion}

\section{Fluorescence and Irradiation Experiments}

The details on the synthesis of $\mathbf{2}$ and $\mathbf{3}$ and the description of photochemical experiments are given in the Supporting Information (SI).

Synthesis and photochemistry of $\mathbf{2}$ has been reported by Eguchi et al..$^{28}$ Photochemistry of $\mathbf{3}^{10-23}$ and a number of alkyl phenyl carbene precursors has been investigated, ${ }^{29,30}$ but no evidence of anti-Kasha photochemistry or fluorescence from higher excited states have been reported. We also investigated photophysics of $\mathbf{2}$ and $\mathbf{3}$, where $\mathbf{2}$ showed unexpected fluorescence properties (Figs S1-S5 and Table S2 in the SI). Due to explosive character, 1 was investigated only computationally. 
Fig. 1 shows absorption and fluorescence spectra of $\mathbf{2}$ measured in benzene. Spectra measured in hexane, cyclohexane and benzene are reported in the SI. The diazo compound is characterized by two absorption bands. The first band is weak $(\varepsilon \approx 30-50$ $\left.\mathrm{M}^{-1} \mathrm{~cm}^{-1}\right)$ and centered at $500 \mathrm{~nm}$ (characteristic pink color), whereas the second is intense $\left(\varepsilon \approx 11000 \mathrm{M}^{-1} \mathrm{~cm}^{-1}\right)$ and centered at $\approx 300 \mathrm{~nm}$. The diazo compound is fluorescent when excited to $\mathrm{S}_{\mathrm{n}}$ with the quantum yield of $\Phi_{\mathrm{F}} \approx 0.04$. Upon excitation to $\mathrm{S}_{1}$ fluorescence could not be detected.
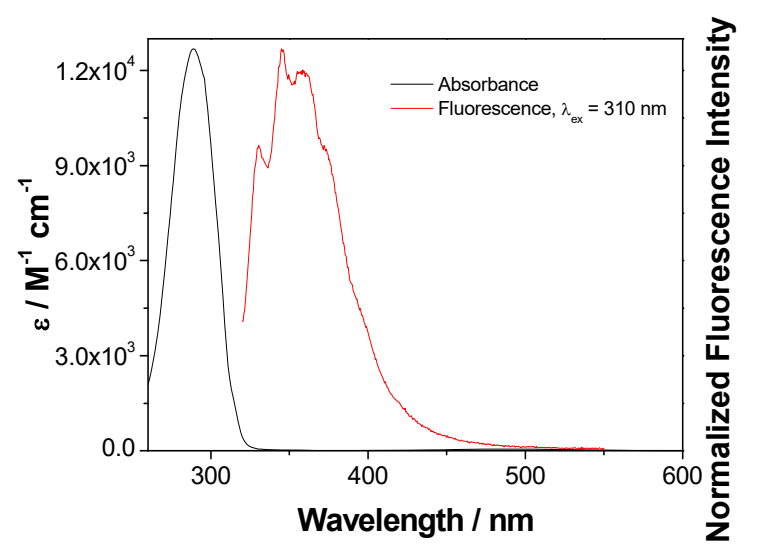

Figure 1. Absorption spectrum for $\mathbf{2}$ and normalized fluorescence spectrum $\left(\lambda_{\mathrm{ex}}=310 \mathrm{~nm}\right)$ in benzene. See Fig. S2 for comparison with computed absorption spectrum.

Prompted by the observation of fluorescence of $\mathbf{2}$ from a higher excited state, we investigated if the molecule shows anti-Kasha photochemistry. Preparative irradiations for $\mathbf{2}$ and $\mathbf{3}$ were performed in cyclohexane and benzene upon excitation with visible light (cool white lamps, corresponding to the population of $\mathrm{S}_{1}$ ) and at $\approx 300 \mathrm{~nm}$ giving rise to $\mathrm{S}_{\mathrm{n}}$ (Schemes $\mathrm{S} 3-\mathrm{S} 8$ and Table $\mathrm{S} 1$ in the $\mathrm{SI})$.

Table 1. Quantum yields $\left(\Phi_{\mathrm{R}}\right)$ for the photoelimination of nitrogen from 2 and $3 .^{\mathrm{a}}$

\begin{tabular}{|c|c|c|}
\hline Compound / Solvent & $\Phi_{300^{\mathrm{b}, \mathrm{c}}}$ & $\Phi_{500^{\mathrm{b}, \mathrm{d}}}$ \\
\hline $\mathbf{2}$ / cyclohexane & $0.48 \pm 0.04$ & $0.048 \pm 0.001$ \\
\hline $\mathbf{2}$ / benzene & $0.49 \pm 0.02$ & $0.010 \pm 0.001$ \\
\hline $\mathbf{3}$ / cyclohexane & $0.36 \pm 0.01$ & $0.020 \pm 0.001$ \\
\hline $\mathbf{3}$ / benzene & $0.46 \pm 0.06$ & $0.020 \pm 0.001$ \\
\hline
\end{tabular}

${ }^{a}$ Measurement were conducted by irradiating the samples at 300 $\mathrm{nm}$ or $500 \mathrm{~nm}$ with a use of ferrioxalate actinometer $\left(\Phi_{300}=1.25\right.$, $\left.\Phi_{500}=0.9\right) .{ }^{31}$ Measurements were done in triplicate, the average value is reported and the errors correspond to maximum absolute deviations. ${ }^{\mathrm{b}}$ Quantum yields of compound decomposition, $\Phi_{\mathrm{R}}$, was calculated according to Eqs. S1-S3 in the SI. ${ }^{\mathrm{c}}$ Irradiation time 1 min. ${ }^{\mathrm{d}}$ Irradiation time $4 \mathrm{~h}$.

Since preparative irradiations (Table S1) indicated that photoelimination was more efficient upon excitation at $300 \mathrm{~nm}$ then by use of visible light, we measured quantum yields for the reaction upon excitation to $S_{1}$ and $S_{n}$ (Table 1). The excitation to $\mathrm{S}_{\mathrm{n}}$ leads to 10-50 times more efficient nitrogen elimination then excitation to $S_{1}$, indicating that anti-Kasha photochemistry takes place, which was verified by computations (vide infra).

\section{Computational Methods}

To gain insight into the photochemical mechanisms of $\mathrm{N}_{2}$ extrusion following VIS or UV excitation, we performed extensive $a b$ initio static calculations and trajectory-surface-hopping nonadiabatic dynamics simulations. Owing to the disparity in the molecular size, we opted for an electronic structure method capable of describing 1-3 with the same accuracy. The method of choice is the algebraic diagrammatic construction up to second-order $(\mathrm{ADC}(2))$ method, ${ }^{32-35}$ which is a correlated singlereference method characterized by relatively small mean absolute error $(0.22 \mathrm{eV})$ with respect to benchmark data. ${ }^{36-38}$ The method, implemented in Turbomole, ${ }^{39}$ describes electronically excited states with accuracy similar to the MP2 method for the electronic ground state and has been employed in a number of recent static and dynamics studies of organic molecules. ${ }^{40-42}$ However, system $\mathbf{1}$ is amenable to a more accurate treatment. An extensive investigation of 1 was performed using benchmark extended multi-state complete active space self-consistent field second-order perturbation theory (XMS-CASPT2 ${ }^{43}$ ), as implemented in the BAGEL program package. ${ }^{44,45}$ A detail comparison shows that the ADC(2) method reproduces very accurately the electronic states of $\mathbf{1}$ not only in the Franck-Condon (FC) region, but also along all investigated reaction pathways (see Figures S6-S8) and, hence, can be used to investigate the photochemistry of 1-3. For more details, see the SI.

\section{Static View of the Photoreactions}

Vertical excitation energies of 1-3 are compiled in Table 2. In all three systems the first transition $\left(\mathrm{S}_{0} \rightarrow \mathrm{S}_{1}\right)$ is weak, while the second transition in $\mathbf{1}\left(\mathrm{S}_{0} \rightarrow \mathrm{S}_{2}\right)$ and third transition in $\mathbf{2}$ and $\mathbf{3}$ $\left(\mathrm{S}_{0} \rightarrow \mathrm{S}_{3}\right)$ are characterized by large oscillator strength.

Table 2. Vertical excitation energies in $\mathrm{eV}$ and oscillator strengths (in parenthesis) computed at the XMS(4)CASPT2(12e,10o)/cc-pVDZ (for 1) and ADC(2)/cc-pVDZ (for 1- 3) levels.

\begin{tabular}{|c|c|c|c|c|}
\hline & $\begin{array}{c}\text { XMS(4)- } \\
\text { CASPT2[12e,10o] }\end{array}$ & \multicolumn{3}{|c|}{ ADC(2) } \\
\hline State & $\mathbf{1}$ & $\mathbf{1}$ & $\mathbf{2}$ & $\mathbf{3}$ \\
\hline S1 & 3.03 & 3.42 & 3.05 & 2.82 \\
& $(0.0001)$ & $(0.0001)$ & $(0.0038)$ & $(0.0030)$ \\
\hline S2 & 6.47 & 6.66 & 4.86 & 4.57 \\
& $(0.295)$ & $(0.2789)$ & $(0.0408)$ & $(0.0401)$ \\
\hline S3 & 8.63 & 7.62 & 4.98 & 4.60 \\
& $(0.000)$ & $(0.0170)$ & $(0.1646)$ & $(0.1008)$ \\
\hline
\end{tabular}

From the natural transition orbital (NTO) pairs involved in the excitations and compared in Figure 2, it is evident that the first transition is almost analogous in 1-3. The $\mathrm{S}_{0} \rightarrow \mathrm{S}_{3}$ transition in $\mathbf{2}$ and $\mathbf{3}$ involves translocation of charge to the phenyl ring(s), but locally, at the CNN moiety, bears a strong resemblance to the $\mathrm{S}_{0} \rightarrow \mathrm{S}_{2}$ transition in $\mathbf{1}$. 


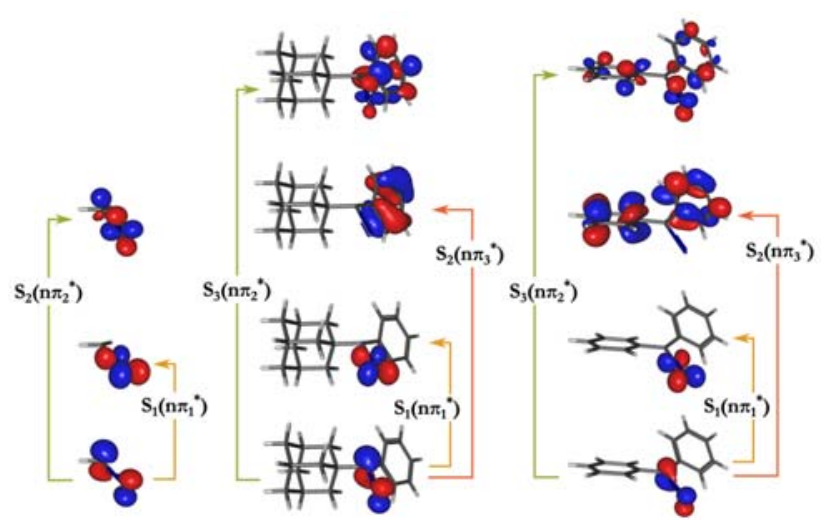

Figure 2. Comparison of dominant natural transition orbital (NTO) pairs describing the lowest singlet excited states of 1-3. Computations have been performed at the ground state equilibrium geometries using the $\mathrm{ADC}(2) / \mathrm{cc}-\mathrm{pVDZ}$ method.

To connect the nature of the initial excitation and the photochemical fate of the system it is useful to consider the difference in the electron densities between the ground and the excited states. ${ }^{46}$ Figure 3 shows that electronic excitation of $\mathbf{1}$ to $S_{1}$ involves translocation of negative charge from the carbon to the nitrogen atoms in the $x y$-plane of the molecule.

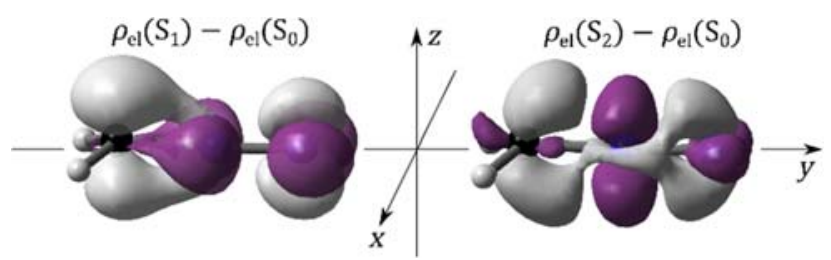

Figure 3. Electron density difference map of 1. Translocation of electron density upon electronic excitation to $S_{1}\left(n \pi_{1}{ }^{*}\right)$ (left) and $\mathrm{S}_{2}\left(\mathrm{n} \pi_{2}{ }^{*}\right)$ (right). Areas of increased (reduced) electron density are shown in pink (silver). The computations have been performed at the $\operatorname{ADC}(2) / c c-p V D Z$ level.

As the nuclei follow the electrons, one can expect that nuclear motion will initially take place in the plane of the molecule. The $\mathrm{S}_{0} \rightarrow \mathrm{S}_{2}$ transition also increases the electron density at the middle $\mathrm{N}$ atom, but it does so perpendicularly to the $x y$-plane of the molecule, suggesting an initial out-of-plane motion of the nitrogen atom. Owing to the comparable character of electronic excitations in 1-3, it is plausible to expect that the initial nuclear motion in the two larger systems will be stirred in the same direction.

Before considering the photochemical reaction paths of 1-3 it is useful to define the three coordinates (see Chart 2) which define the mutual orientation of the $\mathrm{N}_{2}$ and carbene fragments: the C$\mathrm{N}$ distance $R$, the C-N-N bending angle $\varphi$, and the angle between the planes $\sigma_{1}$ and $\sigma_{2}$, denoted $\delta$.

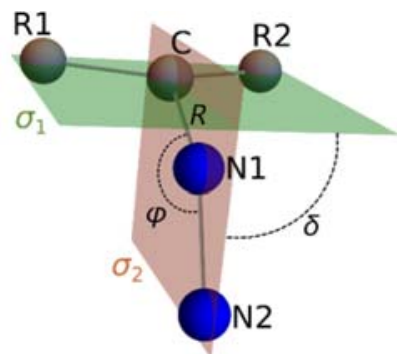

Chart 2. Coordinates describing the mutual orientation of the $\mathrm{N}_{2}$ and R1-C-R2 fragments of 1-3. The C-N distance, $R$, the C-N-N bending angle $\varphi$ (in the $\sigma_{2}$ plane) and the angle $\delta$ between the $\sigma_{1}$ and $\sigma_{2}$ planes. The planes $\sigma_{1}$ and $\sigma_{2}$ are defined by atoms R1-C-R2 and C-N1-N2, respectively.

Excitation of 1-3 to the $S_{1}\left(n \pi_{1}{ }^{*}\right)$ state leads to IC to the ground state. To describe the main features of the reaction we first located relevant CIs, such as the minimum-energy CI geometry $(\mathrm{MECI})^{47}$ and then constructed a linearly interpolated reaction path (LIP) from the Franck-Condon geometry to the MECI. Specifically, we generated a set of molecular geometries by linear interpolation in internal coordinates and performed singlepoint energy calculations for the obtained geometries at the ADC(2) level. Figure 4 shows the ADC(2) energy profiles of the ground state (blue) and the diabatic $n \pi_{1} *$ (orange) and $n \pi_{2} *$ (green) states along the LIPs connecting the Franck-Condon geometries of 1-3 and the corresponding $\mathrm{S}_{1} / \mathrm{S}_{0} \quad \mathrm{CI}$.
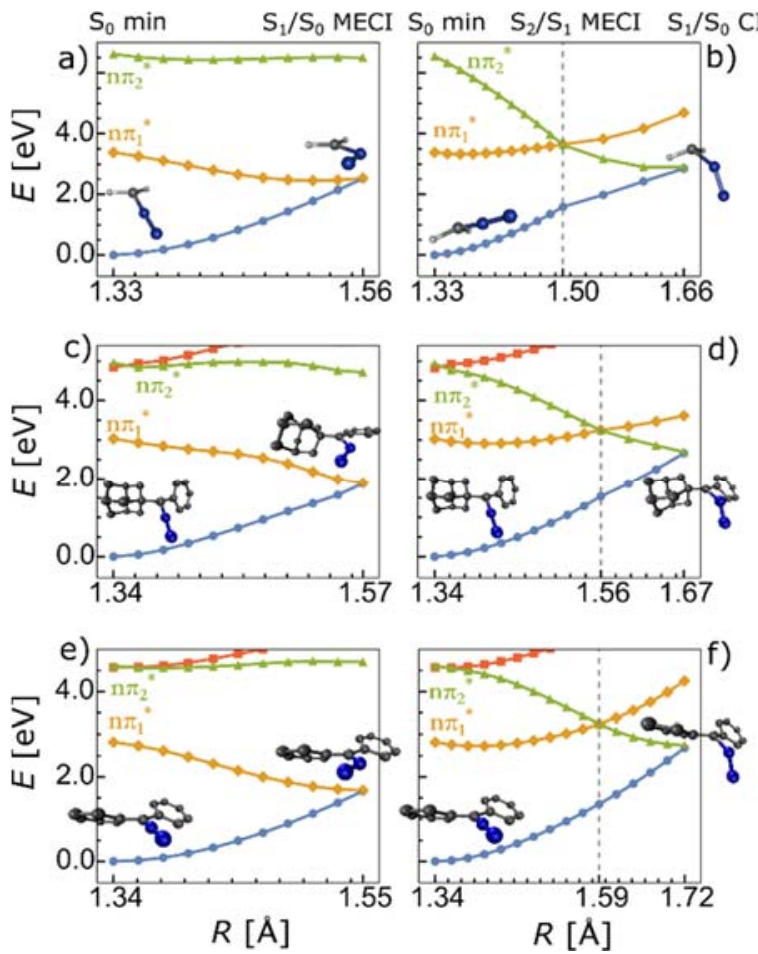

Figure 4. Relative energies of the ground (blue) and diabatic $n \pi_{1}{ }^{*}$ (orange), $\mathrm{n} \pi_{2} *$ (green) and $\mathrm{n} \pi_{3} *$ (red) states of $\mathbf{1}$ (top), $\mathbf{2}$ (middle) and $\mathbf{3}$ (bottom) along the linearly interpolated paths (LIPs) from the FC geometry to the $\mathrm{S}_{1} / \mathrm{S}_{0} \mathrm{CI}$. Left panels show the LIPs from the FC geometry to the $\mathrm{S}_{1}\left(\mathrm{n} \pi_{1} *\right) / \mathrm{S}_{0}$ MECI. Right panels show the LIP from the FC geometry to $\mathrm{S}_{2}\left(\mathrm{n} \pi_{2}{ }^{*}\right) / \mathrm{S}_{1}\left(\mathrm{n} \pi_{1}{ }^{*}\right)$ MECI (vertical line) and to the closest $\mathrm{S}_{1}\left(\mathrm{n} \pi 2^{*}\right) / \mathrm{S}_{0}$ CI. For comparison with the LIPs for 1 computed using the XMS-CASPT2 method see Figure S7. 

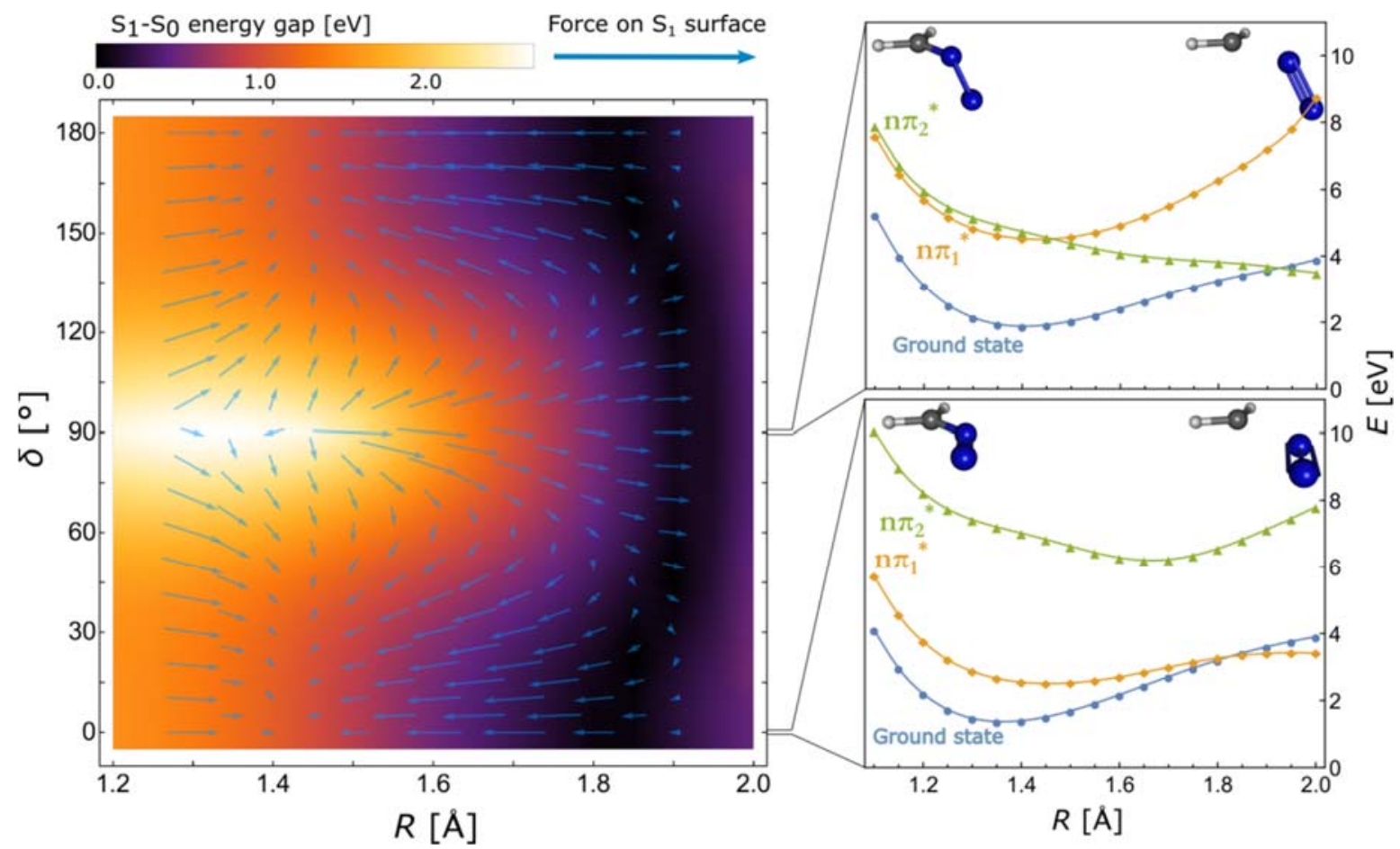

Figure 5. Left: 2D rigid scan of the $\mathrm{S}_{1}-\mathrm{S}_{0}$ energy gap (in $\mathrm{eV}$ ) along the $\mathrm{C}-\mathrm{N}$ distance, $R$, and the angle, $\delta$ (see Chart 2). Superposed blue arrows are scaled force vectors in the $\mathrm{S}_{1}$ state projected to the $(R, \delta)$ subspace, $\left(-\frac{\partial E_{1}}{\partial R},-\frac{\partial E_{1}}{\partial \delta}\right)$. Right: One-dimensional cuts of the three lowest diabatic states for out-of-plane (top) and in-plane (bottom) dissociation. For analogous results computed using the XMS-CASPT2 method see Figure S8.

One immediately sees that all reaction paths are barrierless. Excitation of 1 to the $S_{1}\left(n \pi_{1}^{*}\right)$ state (Figure $4 a$ and Figure S7) leads to the increase of $R$ from 1.33 to $1.56 \AA$ and to the bending of the C-N-N moiety by $\approx-60^{\circ}$, from $176.4^{\circ}$ to $116.8^{\circ}$. As expected on the basis of the translocation of the electron density (Figure 3), dissociation takes place in-the-plane of the molecule. Very similar paths for in-plane $\mathrm{N}_{2}$ extrusion in the larger systems are shown in Figures $4 \mathrm{c}$ and $4 \mathrm{e}$.

On the other side, excitation of $\mathbf{1}$ to $\mathrm{S}_{2}\left(\mathrm{n} \pi_{2}{ }^{*}\right)$ and of $\mathbf{2}$ and $\mathbf{3}$ to $\mathrm{S}_{3}\left(\mathrm{n} \pi_{2}{ }^{*}\right)$ (green) leads to out-of-plane extrusion of $\mathrm{N}_{2}$. The mechanism is shown in the right panels of Figure 4. The first part of the LIP connects the FC geometry and the $\mathrm{S}_{2}\left(\mathrm{n} \pi_{2} *\right) / \mathrm{S}_{1}\left(\mathrm{n} \pi_{1}^{*}\right)$ MECI. These MECIs are non-planar $\left(89.1^{\circ} \leq\right.$ $\left.\delta \leq 90.9^{\circ}\right)$ and characterized by elongated C-N bonds $(1.50 \AA \leq$ $R \leq 1.59 \AA$ ). In the second part of the LIP, a slight elongation of the C-N bond by $0.1-0.16 \AA$, stabilizes the $n \pi_{2}{ }^{*}$ state below the $n \pi_{1}{ }^{*}$ state and leads to the $\mathrm{S}_{1}\left(\mathrm{n} \pi_{2} *\right) / \mathrm{S}_{0} \mathrm{CI}$.

Altogether, two different $\mathrm{S}_{1} / \mathrm{S}_{0}$ CIs exist in 1-3, one between the diabatic $n \pi_{1} *$ state and $S_{0}$ which promotes in-plane fragmentation, and the other, between the diabatic $n \pi_{2}{ }^{*}$ state and $\mathrm{S}_{0}$ leading to out-of-plane fragmentation. The decay through both CIs should lead to the formation of carbene and $\mathrm{N}_{2}$, but the efficiency of the reactions depends on whether the $n \pi_{1}{ }^{*}$ or the $n \pi_{2}{ }^{*}$ state crosses with $\mathrm{S}_{0}$. This point is illustrated in Figure 5 where the two-dimensional (2D) rigid scan shows the change in the energy gap, $\Delta E$, between the $\mathrm{S}_{1}$ and $\mathrm{S}_{0}$ states of 1 with respect to the main reaction coordinates, $R$ and $\delta$ (see Chart 2). The value of $\varphi$ was fixed to $130^{\circ}$. The colour scale (top) indicates the size of the energy gap in $\mathrm{eV}$. One sees that the $\mathrm{S}_{1} / \mathrm{S}_{0} \mathrm{CI}$ seam (black area) is reached along the $\mathrm{C}-\mathrm{N}$ bond dissociation coordinate, $R$. However, the CI seam is significantly curved and reached at shorter $\mathrm{CN}$ distances for in-plane $\left(\delta \approx 0^{\circ}\right.$ and $\left.180^{\circ}\right)$ than for out-of-plane $\left(\delta \approx 90^{\circ}\right)$ dissociation. The two one-dimensional potential energy cuts shown on the right, confirm that out-of-plane $\mathrm{N}_{2}$ extrusion takes place on the $n \pi_{2}{ }^{*}$ while inplane extrusion occurs on the $n \pi_{1} *$ surface. The most important difference, however, lies in the topography of the CIs. The superimposed blue arrows in Figure 5 are the (scaled) force vectors with components $\left(-\frac{\partial E_{1}}{\partial R}\right)$ and $\left(-\frac{\partial E_{1}}{\partial \delta}\right)$. The force vectors were computed analytically ${ }^{39}$ in the $\mathrm{S}_{1}$ state, along which the $\mathrm{S}_{1} / \mathrm{S}_{0} \mathrm{CI}$ seam is approached. The arrows show that for out-ofplane dissociation $\left(\delta \approx 90^{\circ}\right)$ along the diabatic $n \pi_{2} *$ state an outward force acts on the $\mathrm{C}-\mathrm{N}$ bond, leading to an effective separation of the $\mathrm{CH}_{2}$ and $\mathrm{N}_{2}$ fragments. On the contrary, for inplane dissociation in the diabatic $n \pi_{1} *$ state, the forces are directed inward (left arrows) and hinder the separation of the fragments. Figure S8 shows that an analogous scan has been obtained at the XMS-CASPT2 level.

\section{Dynamical View of the Photoreactions}

To verify that the anti-Kasha reactivity of $\mathbf{1 - 3}$ is governed by the same underlying mechanism, we performed non-adiabatic dynamics simulations. For each molecule, 70 trajectories were launched from $\mathrm{S}_{1}$ and from either $\mathrm{S}_{2}$ or $\mathrm{S}_{3}$, depending on the oscillator strength, and propagated until the $\mathrm{S}_{1} / \mathrm{S}_{0}$ CI was reached (for details see S9-S12 in the SI). In all three systems trajectories launched from the $\mathrm{S}_{1}\left(\mathrm{n} \pi_{1} *\right)$ state deactivated to the ground state on the time scale of 50-70 fs. In systems $\mathbf{1}$ and 3, trajectories initiated in the $\mathrm{S}_{2}$ or $\mathrm{S}_{2} / \mathrm{S}_{3}$ states returned to the ground state in 150-200 fs. However, the decay of $\mathbf{2}$ is slower and the ground state was completely repopulated only after 450 fs. The slower decay of $\mathbf{2}$ after photoexcitation to $\mathrm{S}_{2} / \mathrm{S}_{3}$ indicates that the $S_{2}\left(n \pi_{2}{ }^{*}\right)$ surface is rather flat. In solution, where the excess kinetic energy can be redistributed to the solvent on the time scale of around $1 \mathrm{ps}$, this may result in a partial trapping of 
population in $\mathrm{S}_{2}$ and could potentially explain the weak fluorescence from $\mathrm{S}_{2}\left(\Phi_{\mathrm{F}}=0.04\right)$ observed in the experiment (see Figure 1). For comparison of the excited state lifetimes of 1-3 see Figure S9 and S10.

The most significant difference between the simulations initialized in the $S_{1}$ and $S_{2}$ states concerns the geometries of the $S_{1} / S_{0}$ CIs. In all three systems, trajectories initialized in the $\mathrm{S}_{1}$ state reached the ground state via a planar $\left(\delta \approx 0^{\circ}\right.$ or $\left.180^{\circ}\right) \mathrm{S}_{1} / \mathrm{S}_{0} \mathrm{CIs}$, while those initiated in $\mathrm{S}_{2}\left(\mathrm{~S}_{3}\right)$ deactivated mostly via non-planar $\mathrm{S}_{1} / \mathrm{S}_{0}$ CIs. The results are summarized in Figure 6 where the
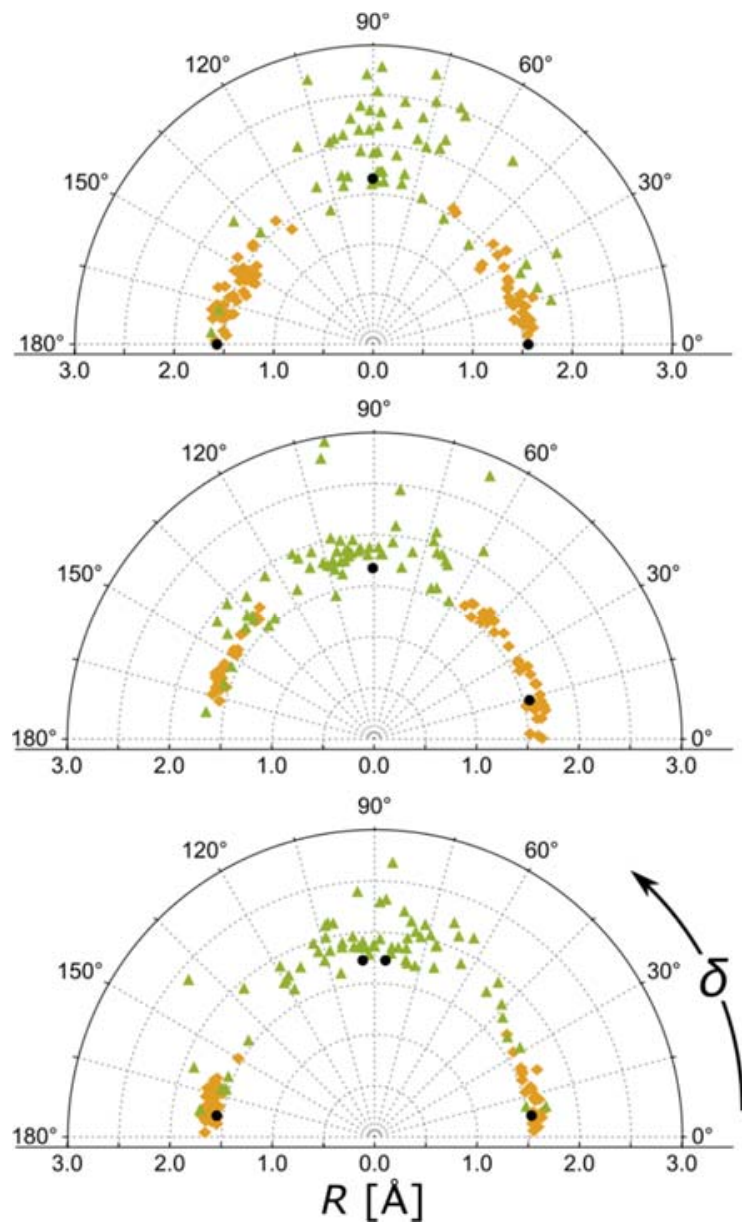

Figure 6. 2D projections of a set of structures of 1 (top), $\mathbf{2}$ (center) and 3 (bottom) in the vicinity of the $\mathrm{S}_{1} / \mathrm{S}_{0} \mathrm{CI}$ seam on the $(R, \delta)$ subspace. Points originate from trajectories initialized in $\mathrm{S}_{1}$ (orange) and in $\mathrm{S}_{2} / \mathrm{S}_{3}$ (green). Black points indicate the $\mathrm{S}_{1}\left(\mathrm{n} \pi_{1}{ }^{*}\right) / \mathrm{S}_{0}$ MECIs $\left(\delta \approx 0^{\circ}\right.$ and $\left.180^{\circ}\right)$ and $\mathrm{S}_{1}\left(\mathrm{n} \pi_{2} *\right) / \mathrm{S}_{0}$ minimal distance CIs $(\delta$ $\approx 90^{\circ}$ ). Nonadiabatic dynamics simulations were performed at the $\operatorname{ADC}(2) / c c-p V D Z$ level.

projections of the geometries belonging to the $\mathrm{S}_{1} / \mathrm{S}_{0} \mathrm{CI}$ seam on the 2D subspace spanned by the C-N distance $R$ and the angle $\delta$ are shown. It is evident that the topography of the $\mathrm{S}_{1} / \mathrm{S}_{0} \mathrm{CI}$ seam of 1-3 is very similar. Compared to the Franck-Condon geometry, all structures have elongated $\mathrm{CN}$ bonds, but the elongation is significantly larger for out-of-plane $\mathrm{N}_{2}$ elimination $\left(\delta \approx 90^{\circ}\right)$ and this happens only if the dynamics is initiated from a higher excited state (green).

As a side result, let us mention that trajectories of $\mathbf{1}$ computed using the XMS-CASPT2 method and initiated in $\mathrm{S}_{1}\left(\mathrm{n} \pi_{1}{ }^{*}\right)$ were naturally continued in the ground state (for characteristic trajectories see Figures S11 and S12). In $\sim 1 / 3$ of trajectories diazirine was formed from a hot ground state of diazomethane confirming the 1994 prediction of Yamamoto et al. ${ }^{24}$

\section{Conclusion}

This work provides evidence of violation of the Kasha rule in the formation of carbenes from diazoalkanes 1-3. We have shown that diazomethane derivatives 1-3 undergo photochemical formation of carbenes and $\mathrm{N}_{2}$ by the same mechanism, where excitation to higher-lying excited singlet states is necessary for the elimination to take place efficiently. Excitation to $\mathrm{S}_{1}\left(\mathrm{n} \pi_{1} *\right)$ leads to in-plane deactivation in a segment of the $\mathrm{S}_{1}\left(\mathrm{n} \pi_{1}{ }^{*}\right) / \mathrm{S}_{0}$ CI seam where the forces acting on the systems hinder the separation of the carbene and $\mathrm{N}_{2}$ fragments. In contrast, excitation to $S_{2}\left(S_{2} / S_{3}\right)$ for $\mathbf{1}(\mathbf{2}-3)$ leads to non-planar CIs and results in about 10-50 times more efficient $\mathrm{N}_{2}$ elimination.

\section{ASSOCIATED CONTENT}

Supporting Information contains details on synthesis and photochemistry of $\mathbf{2}$ and $\mathbf{3}$, photophysical properties of $\mathbf{2}$, computational details including molecular coordinates and copies of ${ }^{1} \mathrm{H}$ and ${ }^{13} \mathrm{C}$ NMR spectra of all compounds. This material is available free of charge via the Internet at http://pubs.acs.org.

\section{AUTHOR INFORMATION}

\section{Corresponding Author}

* ND E-mail: Nadja.Doslic@irb.hr; NB E-mail nbasaric@irb.hr.

\section{Author Contributions}

The manuscript was written through contributions of all authors. MA and TP are both first authors who contributed equally.

\section{Funding Sources}

Croatian Science Foundation (HRZZ IP-2014-09-6312 and IP2016-06-1142).

\section{ACKNOWLEDGMENT}

These materials are based on work financed by the Croatian Science Foundation (HRZZ IP-2014-09-6312 and IP-2016-06-1142). The authors acknowledge generous support of Professors Peter Wan and Cornelia Bohne from the University of Vicoria, Canada $\mathrm{BC}$ and Centre for Advanced Materials and Related Technologies (CAMTEC), University of Victoria, Canada BC for access to the equipment. We thank our team members D. Babić, A. Ponzi and M. Sapunar for constructive discussions and suggestions.

\section{REFERENCES}

1. Demchenko, A. P.; Tomin, V. I. Chou, P. T., Breaking the Kasha Rule for More Efficient Photochemistry, Chem. Rev. 2017, 117, 13353-13381.

2. Voskresenska, V.; Wilson, R. M.; Panov, M.; Tarnovsky, A. N.; Krause, J. A.; Vyas, S.; Winter, A. H.; Hadad, C. M., Photoaffinity Labeling via Nitrenium Ion Chemistry: Protonation of the Nitrene Derived from 4-Amino-3-nitrophenyl Azide to Afford Reactive Nitrenium Ion Pairs, J. Am. Chem. Soc. 2009, 131, 11535-11547.

3. Kubicki, J.; Zhang, Y.; Vyas, S.; Burdzinski, G.; Luk, H. L.; Wang, J.; Xue, J.; Peng, H.-L.; Pritchina, E. A.; Sliwa, M.; Buntinx, G.; Gritsan, N. P.; Hadad, C. M.; Platz, M. S., Photochemistry of 2-Naphthoyl Azides. An Ultrafast Time-Resolved 
UV-Vis and IR Spectroscopic and Computational Study, J. Am. Chem. Soc. 2011, 133, 9751-9761.

4. Burdzinski, G.; Hackett, J. C; Wang, J.; Gustafson, T. L.; Hadad, C. M.; Platz, M. S., Early Events in the Photochemistry of Aryl Azides from Femtosecond UV/Vis Spectroscopy and Quantum Chemical Calculations, J. Am. Chem. Soc. 2006, 128, 13402-13411.

5. Maas, G., New Syntheses of Diazo Compounds, Angew. Chem. Int. Ed. 2009, 48, 8186-8195.

6. Abdel-Wahab, A.-M. A.; Ahmed, S. A.; Dürr, H., Carbene Formation by Extrusion of Nitrogen, in CRC Handbook of Photochemistry and Photobiology 2nd Ed., (Eds.: Horspool, W.; Lenci, F.), CRC Press, Boca Raton, 2004.

7. Black, T. H., The Preparation and Reactions of Diazomethane, Aldrichimica Acta, 1983, 16, 1-22.

8. Holland, J. P.; Gut, M.; Klingler, S.; Fay, R.; Guillou, A., Photochemical Reactions in the Synthesis of Protein-Drug Conjugates, Chem. Eur. J. 2020, 26, 33-48.

9. Nakamoto, K.; Ueno, Y.; Diazirine-Containing RNA Photo-Cross-Linking Probes for Capturing microRNA Targets, J. Org. Chem. 2014, 79, 2463-2472.

10. Knorr, J.; Sokkar, P.; Costa, P.; Sander, W.; Sanchez-Garcia, E.; Nuernberger, P., How Protic Solvents Determine the Reaction Mechanisms of Diphenylcarbene in Solution, J. Org. Chem. 2019, 84, 11450-11457.

11. Costa, P.; Sander, W., Hydrogen Bonding Switches the Spin State of Diphenylcarbene from Triplet to Singlet, Angew. Chem. Int. Ed. 2014, 53, 5122-5125.

12. Peon, J.; Polshakov, D.; Kohler, B., Solvent Reorganization Controls the Rate of Proton Transfer from Neat Alcohol Solvents to Singlet Diphenylcarbene, J. Am. Chem. Soc. 2002, $124,6428-6438$.

13. Chateauneuf, J. E.; Picosecond Spectroscopic Detection of Diphenylcarbenium Ion in the Photolysis of Diphenyldiazomethane in Aliphatic Alcohols, J. Chem. Soc. Chem. Commun., 1991, 1437-1438.

14. Krimse, W.; Kilian, J., Carbenes and the O-H Bond: Spectroscopic Evidence for Protonation of Diarylcarbenes to Give Diarylcarbenium Ions, J. Am. Chem. Soc. 1990, 112, 63996400.

15. Sander, W. W., Reaction of Diphenylmethylene and Phenylmethylene with Oxygen. A Matrix Isolation Study, J. Org. Chem. 1989, 54, 333-339.

16. Griller, D.; Majewski, M.; McGimpsey, W. G.; Nazran, A. S.; Scaiano, J. C., Reaction of Diphenylcarbene with Diphenyldiazomethane, J. Org. Chem. 1988, 53, 1550-1553.

17. Hadel, L. M.; Maloney, V. M.; P1atz, M. S.; McGimpsey, W. G.; Scaiano, J. C., The Absolute Kinetics of Several Reactions of Substltuted Diphenylcarbenes, J. Phys. Chem. 1986, 90, 2488-2491.

18. Werstiukh, N. H.; Casal, H. L.; Scaiano, J. C.; Reaction of Diphenylcarbene with Oxygen: A Laser Flash Photolysis Study, Can. J. Chem. 1984, 62, 2391-2392.

19. Sitzmann, E.V.; Langan J.G.; Eisenthal, K.B., Picosecond Laser Studies of the Effects of Reactants on Intramolecular Energy Relaxation of Diphenylcarbene: Reaction of Diphenylcarbene with Alcohols, Chem. Phys. Lett. 1984, 112, 111-116.

20. Wright B. B.; Platz, M. S., Chemistry and Kinetics of Aryl Carbenes in Methanol at Low Temperatures, J. Am. Chem. Soc. 1984, 106, 4175-4180.

21. Hadel, L. M.; Platz, M. S.; Scaiano, J. C.; Study of Hydrogen Atom Abstraction Reactions of Triplet Diphenylcarbene in Solution, J. Am. Chem. Soc. 1984, 106, 283-287.
22. Tomioka, H.; Suzuki, S.; Izawa, Y., Carbene in a Rigid Matrix. XII. Temperature and Substituent Effects on Regioselectivity in the Insertion of Arylcarbene into Alcohols, J. Am. Chem. Soc. 1982, 104, 3156-3162.

23. Senthilnathan, V. P.; Platz, M. S., Determination of the Absolute Rates of Decay of Arylcarbenes in Various Low Temperature Matrices by Electron Spin Resonance Spectroscopy, $J$. Am. Chem. Soc. 1980, 102, 7637-7643.

24. Yamamoto, N.; Bernardi, F.; Bottoni, A.; Olivucci, M.; Robb, M. A.; Wilsey, S. Mechanism of Carbene Formation from the Excited States of Diazirine and Diazomethane: An MC-SCF Study, J. Am. Chem. Soc. 1994, 116, 2064-2074.

25. Arenas, J. F.; Lopez-Tocon, I.; Otero, J. C.; Soto, J. Carbene Formation in Its Lower Singlet State from Photoexcited 3H-Diazirine or Diazomethane. A Combined CASPT2 and ab Initio Direct Dynamics Trajectory Study. J. Am. Chem. Soc. 2002, 124, 1728-1735.

26. Li, Q.; Migani, A.; Blancafort, L., Wave Packet Dynamics at an Extended Seam of Conical Intersection: Mechanism of the Light-Induced Wolff Rearrangement, J. Phys. Chem. Lett. 2012, 3, 1056-1061.

27. Li, H.; Migani, A.; Blancafort, L.; Li, Q.; Li, Z., Early Events in the Photochemistry of 5-Diazo Meldrum's Acid: Formation of a Product Manifold in C-N Bound and Pre-dissociated Intersection Seam Regions, Phys. Chem. Chem. Phys. 2016, 18, 30785-30793.

28. Ohno, M.; Itoh, M.; Umeda, M.; Furuta, R.; Kondo, K.; Eguchi, S., Conjugatively Stabilized Bridgehead Olefins: Formation and Reaction of Remarkably Stable Homoadamant-3enes Substituted with Phenyl and Methoxycarbonyl Groups, $J$. Am. Chem. Soc. 1996, 118, 7075-7082.

29. Wang, J.; Burdzinski, G.; Gustafson, T. L.; Platz, M. S., Ultrafast Study of p-Biphenylyldiazoethane. The Chemistry of the Diazo Excited State and the Relaxed Carbene, J. Am. Chem. Soc. 2007, 129, 2597-2606.

30. Motschiedler, K.; Gudmundsdottir, A.; Toscano, J. P.; Platz, M.; Garcia-Garibay, M. A., Excited Precursor Reactivity, Fast 1,2-H Shifts, and Diffusion-Controlled Methanol Insertion in 1,2-Diphenylalkylidenes, J. Org. Chem. 1999, 64, 51395147.

31. H. J. Kuhn, S. E. Braslavsky and R. Schmidt, Chemical Actinometry (IUPAC Technical Report), Pure Appl. Chem. 2004, 76, 2105-2146.

32. Schirmer, J. Beyond the Random-Phase Approximation: A New Approximation Scheme for the Polarization Propagator, Phys. Rev. A 1982, 26, 2395-2416.

33. Trofimov, A. B.; Schirmer, J. Polarization Propagator Study of Electronic Excitation in key Heterocyclic Molecules I. Pyrrole, Chem. Phys. 1997, 214, 153-170.

34. Wormit, M.; Rehn, D. R.; Harbach, P. H.; Wenzel, J.; Krauter, C. M.; Epifanovsky, E.; Dreuw, A. Investigating Excited Electronic States Using the Algebraic Diagrammatic Construction (ADC) Approach of the Polarisation Propagator, Mol. Phys. 2014, 112, 774-784.

35. Dreuw, A.; Wormit, M. The Algebraic Diagrammatic Construction Scheme for the Polarization Propagator for the Calculation of Excited States, WIREs Comput. Mol. Sci. 2015, 5, 82-95.

36. Schreiber, M.; Silva-Junior, M. R.; Sauer, S. P. A.; Thiel, W., Benchmarks for Electronically Excited States: CASPT2, CC2, CCSD, and CC3, J. Chem. Phys. 2008, 128, 134110134125. 
37. Sauer, S. P. A.; Schreiber, M.; Silva-Junior, M. R.; Thiel, W., Benchmarks for Electronically Excited States: A Comparison of Noniterative and Iterative Triples Corrections in Linear Response Coupled Cluster Methods: CCSDR(3) versus CC3, $J$. Chem. Theory Comput. 2009, 5, 555-564.

38. Winter, N. O. C.; Graf, N. K.; Leutwyler, S.; Hättig, C., Benchmarks for 0-0 Transitions of Aromatic Organic Molecules: DFT/B3LYP, ADC(2), CC2, SOS-CC2 and SCS-CC2 Compared to High-Resolution Gas-Phase Data, Phys. Chem. Chem. Phys. 2013, 15, 6623-6630.

39. Furche, F.; Ahlrichs, R.; Hättig, C.; Klopper, W.; Sierka, M.; Weigend, F. Turbomole. Wiley Interdiscip. Rev.: Comput. Mol. Sci. 2014, 4, 91-100.

40. Prlj, A.; Curchod, B. F. E.; Corminboeuf, C., Excited State Dynamics of Thiophene and Bithiophene: New Insights into Theoretically Challenging Systems, Phys. Chem. Chem. Phys. 2015, 17, 14719-14730.

41. Novak, J.; Prlj, A.; Basarić, N.; Corminboeuf, C.; Došlić, N., Photochemistry of 1- and 2-Naphthols and Their Water Clusters: The Role of ${ }^{1} \pi \pi^{*}\left(\mathrm{La}_{\mathrm{a}}\right)$ Mediated Hydrogen Transfer to Carbon Atoms, Chem. Eur. J. 2017, 23, 8244-8251.
42. De Vetta, M.; González, L.; Corral, I., The Role of Electronic Triplet States and High-Lying Singlet States in the Deactivation Mechanism of the Parent BODIPY: An ADC(2) and CASPT2 Study, ChemPhotoChem, 2019, 3, 727-738.

44. Shiozaki, T.; Gyorffy, W.; Celani, P.; Werner, H-J. Extended Multi-State Complete Active Space Second-Order Perturbation Theory: Energy and Nuclear Gradients. J. Chem. Phys. 2011, 135, 081106.

44. Shiozaki, T. BAGEL: Brilliantly Advanced General Electronic-structure Library. WIREs Comput. Mol. Sci. 2018, 8, No. el1311.

45. Shiozaki, Toru, BAGEL: Brilliantly Advanced General Electronic-structure Library. http://www.nubakery.org, under the GNU General Public License.

46. Zimmerman, H. E.; Alabugin, I. V., Excited State Energy Distribution and Redistribution and Chemical Reactivity; Mechanistic and Exploratory Organic Photochemistry, J. Am. Chem. Soc. 2000, 122, 952-953.

47. Levine, B. G.; Coe, J. D.; Martínez, T. J., Optimizing Conical Intersections without Derivative Coupling Vectors: Application to Multistate Multireference Second-Order Perturbation Theory (MSCASPT2), J. Phys. Chem. B 2008, 112, 405413. 


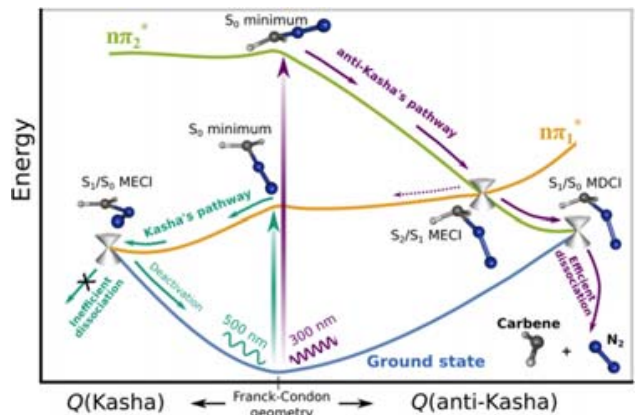

